\title{
Frontières
}

\section{Adaptation et résilience}

\section{Favoriser la recherche de sens chez les personnes atteintes de sclérose en plaques}

\section{Christiane Couture}

Volume 22, numéro 1-2, automne-printemps 2009-2010

Résilience et deuil

URI : https://id.erudit.org/iderudit/045024ar

DOI : https://doi.org/10.7202/045024ar

Aller au sommaire du numéro

\section{Éditeur(s)}

Université du Québec à Montréal

ISSN

1180-3479 (imprimé)

1916-0976 (numérique)

Découvrir la revue

Citer cet article

Couture, C. (2009). Adaptation et résilience : favoriser la recherche de sens chez les personnes atteintes de sclérose en plaques. Frontières, 22(1-2), 27-34.

https://doi.org/10.7202/045024ar

\section{Résumé de l'article}

S'adapter à la sclérose en plaques (SEP) constitue un défi renouvelé au quotidien. La conceptualisation du processus d'adaptation pour les personnes atteintes de SEP à partir des théories sur le deuil permet une réflexion ouvrant sur des pistes d'intervention. Les caractéristiques spécifiques de l'adaptation à la SEP sont décrites pour faire le lien entre les principales étapes du processus, ses dérives et ses impasses qui sont définies comme des troubles de l'adaptation. Selon la littérature récente, le sens est déterminant dans l'adaptation réussie et la résilience. Des études tendent à démontrer que les troubles d'adaptation chez les personnes atteintes de SEP sont moins fréquents lorsqu'elles parviennent à donner un sens à leur maladie. D'où la pertinence de faciliter l'adaptation par la recherche de sens. La présentation d'une activité de groupe illustre une telle intervention en réadaptation.
Ce document est protégé par la loi sur le droit d'auteur. L'utilisation des services d’Érudit (y compris la reproduction) est assujettie à sa politique d'utilisation que vous pouvez consulter en ligne.

https://apropos.erudit.org/fr/usagers/politique-dutilisation/ 


\section{Résumé}

S'adapter à la sclérose en plaques (SEP) constitue un défi renouvelé au quotidien. La conceptualisation du processus d'adaptation pour les personnes atteintes de SEP à partir des théories sur le deuil permet une réflexion ouvrant sur des pistes $d$ 'intervention. Les caractéristiques spécifiques de l'adaptation à la SEP sont décrites pour faire le lien entre les principales étapes du processus, ses dérives et ses impasses qui sont définies comme des troubles de l'adaptation. Selon la littérature récente, le sens est déterminant dans l'adaptation réussie et la résilience. Des études tendent à démontrer que les troubles d'adaptation chez les personnes atteintes de SEP sont moins fréquents lorsqu'elles parviennent à donner un sens à leur maladie. D'où la pertinence de faciliter l'adaptation par la recherche de sens. La présentation d'une activité de groupe illustre une telle intervention en réadaptation.

Mots clés: sclérose en plaques adaptation - deuil - recherche de sens résilience.

\section{Abstract}

Coping with multiple sclerosis (MS) is a challenge that must be faced every day. Conceptualizing the coping process for people with multiple sclerosis (MS) based on bereavement theories provides for reflection that leads to possible interventions. The specific characteristics of coping with MS are described so as to establish a connection between the principal steps in the process, its derivatives and its impasses, which are defined as coping problems. According to recent literature, meaning is a decisive factor in the case of successful coping and resilience. Studies tend to demonstrate that people with MS experience fewer coping problems when they manage to give meaning to their disease. As a result, facilitating coping through a search for meaning is pertinent. A group activity is presented to illustrate such a rehabilitation intervention.

Keywords: multiple sclerosis - coping bereavement - search for meaning resilience.

\section{ADAPTATION ET RÉSILIENCE \\ Favoriser la recherche de sens chez les personnes atteintes de sclérose en plaques}

\begin{abstract}
Christiane Couture, M.Ps., psychologue, Programme des maladies neurologiques évolutives, Centre de réadaptation Lucie-Bruneau.
\end{abstract}

\section{LA SCLÉROSE EN PLAQUES}

La sclérose en plaques (SEP) est la maladie neurologique la plus fréquente chez les adultes qui frappe principalement les gens âgés entre 15 et 40 ans. Au Canada, sa prévalence est de 240 cas pour 100000 habitants. De 13000 à 18000 Québécoises et Québécois en sont atteints dans une proportion de plus de deux femmes pour un homme (Société canadienne de sclérose en plaques, 2009). Décrite initialement par Charcot en 1873, il s'agit d'une maladie auto-immune qui entrave la conduction nerveuse en détruisant la gaine de myéline entourant les nerfs et pouvant provoquer éventuellement une perte axonale. L'étiologie en est encore mal comprise. L'évolution se fait par poussées-rémissions ou peut prendre une forme progressive. Le pronostic en est imprévisible. L'inflammation laisse des cicatrices dans le système nerveux qui donnent des symptômes différents selon leur localisation. Les troubles visuels et urinaires sont fréquents mais aussi les déficits moteurs, cérébelleux et sensitifs. La fatigue en est le symptôme le plus courant et parfois le plus handicapant (Société canadienne de sclérose en plaques, 2009). Une fatigue sévère est d'ailleurs rapportée par $52 \%$ à $93 \%$ des personnes diagnostiquées (Boërio et al., 2006). Les atteintes cognitives sont présentes dans $40 \%$ à $70 \%$ des cas; elles se manifestent principalement par le ralentissement du traitement de l'information, les problèmes de mémoire récente, autant verbale que visuelle, les atteintes des fonctions exécutives et du traitement des données visuospatiales (Diaz-Olavarrieta et al., 1999). La capacité d'attention, soutenue et divisée, est fragile (Rogers et Panegyres, 2007). Les capacités verbales sont généralement mieux préservées, quoique le manque du mot soit fréquent, ce qui peut conduire à une sous-évaluation des limitations cognitives. 


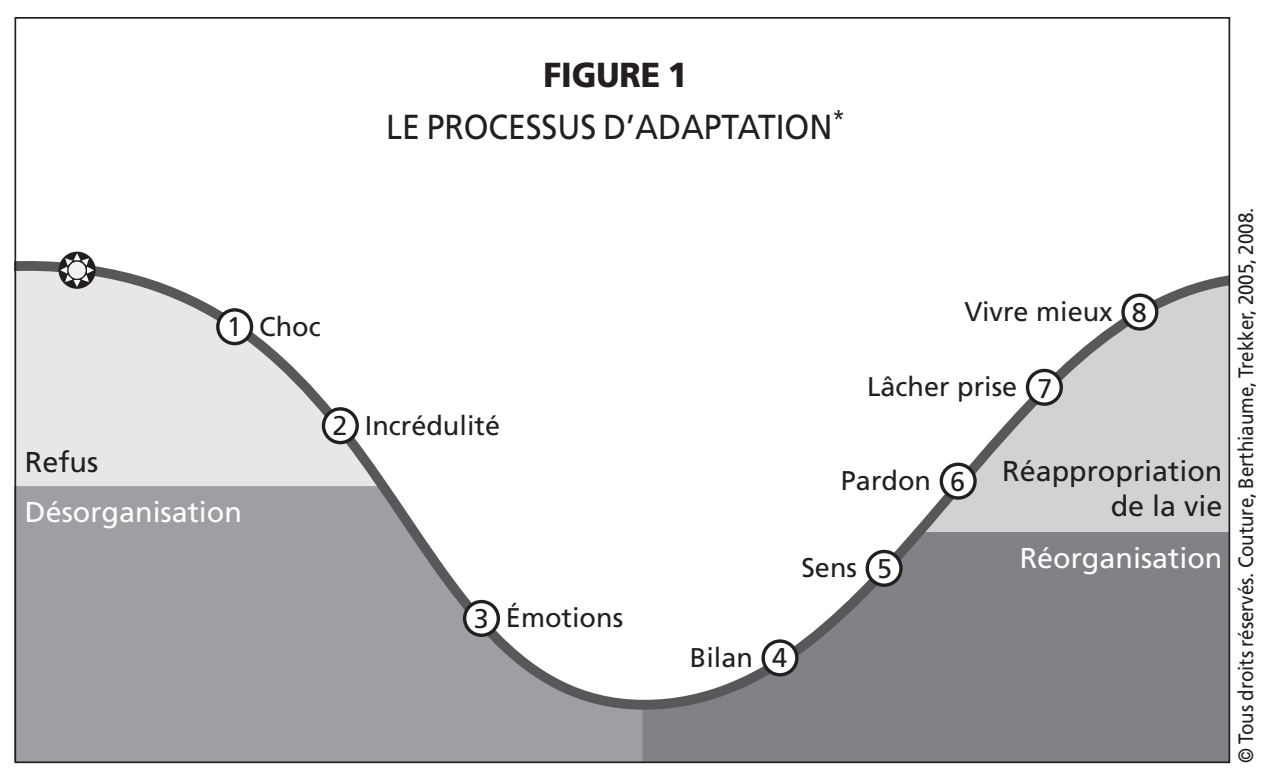

* Inspiré de Monbourquette (1983) ; Lemieux (2002); St-Germain (1996).

Chez les gens atteints de SEP, les manifestations psychopathologiques sont beaucoup plus fréquentes que dans la population en général. Il est entendu que certains symptômes de la SEP peuvent être confondus avec des signes de troubles de santé mentale comme la fatigue, les troubles cognitifs, les tremblements. Tout de même, les psychoses et les troubles bipolaires sont deux fois plus fréquents que dans la population en général, l'euphorie est observable chez 9 à 13\%, la dépression est présente chez environ $20 \%$ et l'anxiété chez plus de 15\% (Chwastiak et Ehde, 2007). Plusieurs auteurs comprennent cette forte comorbidité comme une indication endogène: la SEP elle-même, par ses lésions au système nerveux central, créerait aussi les manifestations psychiatriques (Beiske et al., 2008). Une autre hypothèse, psychosociale celle-là, met en évidence la réaction aux stress multiples engendrés par l'évolution de la maladie pour comprendre les réactions émotives (Goldman Consensus Group, 2005) et même la survenue des poussées de SEP (Buljevac et al., 2003).

Vivre avec la sclérose en plaques (SEP) constitue donc un défi renouvelé au quotidien. Non seulement l'évolution de la maladie est-elle imprévisible, les pertes multiples et l'état variable, encore faut-il s'attendre à une succession de changements qui peuvent survenir à un rythme qui dépasse les capacités d'adaptation. Sans oublier que les proches sont aussi affectés par la maladie. De plus, dans un contexte culturel où la performance et la réussite sont souvent des bases importantes de l'identité, la maladie chronique, évolutive de surcroît, constitue une épreuve à l'âge adulte.
Compte tenu des caractéristiques reliées à la SEP, qu'en est-il des questions d'adaptation, de résilience et de deuil? Le présent texte propose quelques réponses à ces questions d'un point de vue théorique et clinique. Le processus d'adaptation, dans sa compréhension empruntée du deuil, sera d'abord présenté avant de le considérer dans ses caractéristiques liées à une maladie évolutive. Les impasses de l'adaptation seront ensuite abordées: deuil compliqué et deuil pathologique. La résilience sera abordée sous l'angle de l'adaptation réussie et de la recherche de sens avant d'appliquer ces concepts à la SEP. Un exemple d'intervention clinique en réadaptation sera offert en terminant sur les défis que présente le soutien aux personnes atteintes de SEP et leurs proches.

\section{L'ADAPTATION}

Conceptualisé à partir des théories sur le deuil, le processus d'adaptation est expliqué dans un modèle linéaire comme un enchaînement d'étapes. Comme l'illustre la figure 1, au fil de l'expérience clinique, le vocabulaire a été adapté à la sensibilité et à la compréhension du phénomène tel qu'il est expérimenté par les personnes atteintes de SEP qui ont réagi, parfois avec véhémence, en comparant leur expérience au discours théorique qui leur était présenté. Le deuil leur est expliqué comme une réaction progressive naturelle d'accommodation à une situation qui provoque une remise en question de leur équilibre habituel. L'ampleur de la réaction est en lien avec l'importance de ce qui est perdu. Les circonstances de la perte peuvent avoir une influence sur la façon dont le deuil va se dérouler. Freud (1986) déjà considérait le deuil comme la réaction graduelle à l'épreuve de réalité de la perte, perte d'objet interne, y compris une partie de soi.

S'adapter à la SEP, c'est faire face à la perte graduelle de son intégrité physique et parfois cognitive, de ses rôles sociaux. La SEP, comme d'autres conditions médicales, engendre une reconstruction identitaire chez la personne atteinte mais aussi chez ses proches (Saint-Charles, 1998; Lefebvre et Levert, 2005). Il peut être intéressant de noter que le présent modèle, construit initialement pour les personnes atteintes de SEP, a été soumis à leurs proches et à des personnes atteintes de sclérose latérale amyotrophique ou d'encéphalopathies qui en ont aussi reconnu la pertinence pour eux.

\section{ÉTAPES DU PROCESSUS D'ADAPTATION}

Selon les auteurs consultés, l'adaptation se fait en quatre phases (Lemieux, 2002), en huit étapes (Monbourquette, 1994; Saint-Germain, 1996) ou plus encore (Tremblay, 2001; Lefebvre et Levert, 2005). Quoi qu'il en soit, tous s'entendent pour dire que, dans un premier temps, la personne refuse le changement et tente de retourner à l'état initial pour éviter la désorganisation que va entraîner la nouvelle situation. Mais la réalité de la perte s'impose et avec elle, la personne va entreprendre de se réorganiser avant de se recréer une nouvelle existence.

LA PHASE DE NÉGATION CONSTITUE UNE TENTATIVE DE REFUSER LA SOUFFRANCE QUI SE DISTINGUE EN DEUXTEMPS.

D'abord l'état de choc s'installe, qui est une défense psychologique pour ne pas s'effondrer. Cette réaction initiale d'hébétude, une sorte d'engourdissement émotif, sert à protéger la personne de l'ampleur du déséquilibre induit par le changement.

Puis l'incrédulité survient. Il s'agit aussi d'une stratégie active de négation de la réalité qui se manifeste de différentes façons (Pitzele, 2000). Sur un plan cognitif, par l'oubli de l'information, l'évitement des lieux et des circonstances rappelant la perte, comme fuir la vue du fauteuil roulant. $\mathrm{Ou}$ au contraire par le rappel constant de ce qui a été perdu, l'étalage de photos et autres objets qui rappellent le «bon vieux temps», un discours répétitif sur cet âge d'or antérieur. Chez ceux qui ne se permettent pas d'avoir d'émotions, la négation se fait par la substitution, le remplacement rapide par un autre objet, ou la banalisation des conséquences sur sa vie. Mais nier la réalité ne la supprime pas et elle finira par s'imposer d'elle-même. Et alors surviendra la désorganisation. 
LA PHASE DE DÉSORGANISATION

VIENT AVEC LA PRISE DE CONSCIENCE

GRADUELLE DE LA RÉALITÉ DE LA PERTE.

IL S'AGIT ESSENTIELLEMENT

D'UNE ÉTAPE ÉMOTIVE.

Les émotions surgissent lors de la confrontation à la nouvelle réalité qui rend caducs les repères habituels. Elles peuvent être de tout ordre, être changeantes et varier en intensité. La confusion et la désorientation, l'ambivalence sont fréquentes. La sensation de perdre le contrôle peut survenir. La révolte est caractérisée par le «Pourquoi moi?». Elle indique que la personne commence à se mobiliser. La négociation et le marchandage constituent des tentatives de s'en tirer avec moins de contraintes (Keirse, 2000).

Il convient de se rappeler que l'émotion est liée à l'énergie. L'exprimer, comme s'en couper, nolise une partie de nos ressources cognitives comme en témoigne une étude mettant en évidence des déficits mnésiques lorsque l'émotion est sollicitée (Bonanno et al., 2004). L'émotion doit être ressentie, acceptée comme sienne. D'abord se donner le droit d'être émotif, pour ensuite choisir de l'exprimer ou non, et enfin décider sur quelle modalité le faire. Il semble que la flexibilité entre exprimer ou contenir soit un meilleur gage de résolution du deuil que le partage comme tel (Bonanno et al., 2004). L'expression d'émotions, lorsqu'elle est consentie, peut se faire en trouvant quelqu'un avec qui les partager, en les transformant en une activité artistique ${ }^{1}$, en prenant soin de soi.

Ultimement, le sentiment de la perte, la tristesse, met en contact avec l'ampleur de la perte. La pleine conscience permet la reconnaissance des conséquences. La peine profonde qui s'ensuit, sans désorganisation et sans honte, peut nécessiter un repli sur soi permettant de passer aux étapes suivantes. La perte d'intérêt pour le monde extérieur est normale à ce stade (Tremblay, 2001). En cela, le deuil ressemble à la dépression, la perte d'estime de soi en moins (Freud, 1986).

\section{LA PHASE DE RÉORGANISATION}

PERMET DE S'ADAPTER À SON NOUVEL

ENVIRONNEMENT EN DEUX DÉMARCHES

COGNITIVES, CELLES DU BILAN

ET DE LA RECHERCHE DE SENS.

Le bilan consiste à faire l'inventaire; voir ce qu'on perd, ce qu'on gagne. Cette étape permet de faire le point, de clore ce qui doit l'être, de reconnaître ce qui ne sera plus, d'exprimer du regret sans la détresse, d'envisager les risques et les contraintes inhérents aux choix de vie. Kalb (2004) souligne la pertinence de repérer aussi ce qui reste intact (MS free zone) où perdurent l'accalmie de la continuité et le plaisir associé. Le bilan permet enfin de formuler de nouveaux objectifs, de reprendre le fil des activités et des intérêts délaissés, d'en développer de nouveaux. Le risque ici est de jeter le bébé avec l'eau du bain. Pour ne pas renoncer hâtivement à ses anciens centres d'intérêt, la personne atteinte a besoin de procéder à un processus de résolution de problèmes au cours duquel l'équipe de réadaptation propose des alternatives aux anciennes façons de faire. À cette étape, la personne peut commencer à changer ses valeurs et ses priorités, expérimenter les adaptations proposées, appliquer les principes d'économie d'énergie enseignés.

La recherche de sens est une étape cruciale de l'adaptation. Trouver un sens à la perte, c'est répondre aux questions suivantes: Comment la maladie s'inscritelle dans mon histoire de vie? Qu'est-ce que j'ai appris? Qu'est-ce que j'ai changé? Qu'est-ce que je comprends mieux? Quelles sont les ressources que j'ai développées? Comment ma vision des choses a-t-elle changé? Quelle connaissance de moi ai-je acquise? Il convient de voir aussi les deuils secondaires, c'est-à-dire considérer les conséquences de la perte sur le reste de la vie et profiter de l'occasion pour régler les deuils anciens qui refont surface.

Pour certains auteurs, le deuil peut être considéré achevé avec la découverte du sens. Un deuil complété permet un retour de l'énergie et de la créativité, une ouverture au monde. Les prochaines étapes relèvent d'une démarche spirituelle en trois temps.

LA PHASE DE RÉAPPROPRIATION DE LA VIE EST ASSOCIÉE AU FAIT DE REFAIRE LA CONTINUITÉ DE SON EXISTENCE ET DE RÉAPPRENDRE À AIMER LA VIE.

Lefebvre et Levert (2005) nomment cette étape ultime, la transformation. Elles l'illustrent par un processus circulaire en forme de spirale qui rend bien l'effet dynamique et récurrent de l'adaptation.

- Pardonner signifie la fin de la culpabilité et du blâme. D'une part, développer de l'indulgence pour la personne imparfaite que je suis, se pardonner à soi d'être malade, cesser de se considérer comme un fardeau ou de chercher ce qu'on a bien pu faire pour mériter un pareil sort, renoncer à croire qu'on gâche la vie des autres ou qu'on leur impose une réalité qu'ils ne veulent pas. D'autre part, il s'agit aussi de pardonner aux autres d'être en santé, de continuer leur vie en dehors de nous. Pour le détail de cette étape, voir les douze étapes du pardon dans Monbourquette (2007).

- Lâcher prise équivaut à cesser de se battre pour l'inaccessible, se sentir prêt à abandonner l'ancienne image de soi, accepter la réalité actuelle et la façon dont elle s'est installée. Certains se rebiffent à cette idée, qui prend pour eux la connotation de perdre espoir, baisser les bras. «Accepter? Jamais!», disent-ils alors dans un élan amer. Mais tourner la page, renoncer à tout contrôler, n'est pas non plus approuver (Comte-Sponville, 2006).

- Ultimement, la personne est disposée à «vivre mieux». Dans le deuil, cette étape se nomme l'héritage. Elle est la reprise de la continuité de l'histoire et de l'identité personnelles, la projection dans l'avenir. On y est si on peut répondre à la question: Que vais-je faire du reste de ma vie avec les capacités que j'ai développées, avec cette nouvelle personne que je suis devenue?

Le deuil est considéré résolu s'il est envisagé avec un minimum d'émotions et est devenu une occasion de croissance. À ce stade, la personne assume la maladie, son attitude en est une active autant que réaliste. La complétude du processus d'adaptation sonne l'heure de célébrer le changement et de l'approfondir. Quelle cérémonie symbolique peut en consacrer le passage? Peu de rituels existent pour soutenir sa conclusion. D'ailleurs, dans une culture où le déni de la mort est la norme et, avec lui, le rejet de la faiblesse et de la vulnérabilité, la personne qui ne peut guérir dérange. Elle porte la honte de ne pouvoir triompher de ses limites. «Si je veux, je peux», dit l'adage. Si je ne peux pas, je ne vaux rien? Et je suis condamnée à la perte d'estime de soi et à la dépression (Atchley, 1982)? L'anthropologue Michel Desjardins (2002) a mis en évidence la mise en marge sociale subtile des personnes souffrant de déficiences intellectuelles dans notre culture. Les personnes qui ont des limitations physiques ne trouvent pas non plus nécessairement leur place dans la société de consommation et de vitesse où l'on cultive l'espoir de vivre «sans vieillir $»^{2}$. Au contraire, philosophe, lui-même atteint de paralysie cérébrale, Jollien (2002), citant Valéry, clame «je vaux ce que je veux » et fait de son combat au quotidien avec sa condition assumée, «le métier d'homme».

\section{CARACTÉRISTIQUES DU DEUIL LIÉES À UNE MALADIE ÉVOLUTIVE}

La sclérose en plaques présente des caractéristiques spécifiques qui influent sur le processus d'adaptation.

Soulagement. Le diagnostic peut amener un soulagement au lieu du choc initial parce qu'il est la confirmation pour eux et pour l'entourage que quelque chose ne va pas, enfin un savoir concret après tant d'incertitude, la fin du doute sur la santé 
mentale, l'arrêt de l'angoisse associée à des symptômes inexpliqués (Pitzele, 2000).

Déni. La SEP favorise le déni en phase de rémission. Pourquoi faire un deuil quand on peut récupérer?

Imprévisibilité. Comment prévoir à quoi s'adapter quand il n'y a aucune certitude, ni sur la symptomatologie, ni sur le rythme d'évolution, ni sur l'efficacité des traitements?

Spécificité de chaque perte. Chaque perte engendre son propre processus et dépend de la valeur subjective de ce qui a été perdu. Par exemple, le petit doigt de la main gauche pour un pianiste.

Multiplicité. Trop d'adaptations pour une même personne, ou à un rythme trop rapide, peut surcharger la capacité d'adaptation. Voir aussi l'accumulation possible par effet domino: perte fonctionnelle qui résulte en perte d'emploi qui signifie perte de revenu qui entraînera la vente de la maison et, qui sait, l'échec du couple...

Douleur et fatigue. Faire son deuil prend de l'énergie, comme en témoigne l'expression «travail du deuil». Comment faire quand on en manque justement à cause de la fatigue neurologique? La douleur gruge aussi.

Capacité de résolution de problèmes et troubles cognitifs. S'adapter, c'est aussi concrètement régler des problèmes, les uns après les autres. Or, pour une certaine proportion de la clientèle, la SEP limite l'efficacité cognitive.

Les proches des personnes atteintes ont leur propre processus d'adaptation à faire, à leur rythme. Les familles, «ceux qui nous aiment, ceux qui nous aident», sont aussi en deuil (Pitzele, 2000; Lefebvre et Levert, 2005).

Espoir. Il convient de distinguer la nature de l'espoir qui alimente la motivation et la projection vers l'avenir, portée par les progrès de la recherche par exemple, de la pensée magique, qui relève plus du déni, générant des attentes irréalistes et empêchant de s'investir dans l'action concrète au présent (Hétu, 1989). L'espoir fait vivre mais sur la corde raide (Valéry), car le corollaire de l'espoir est la peur (Comte-Sponville, 2006).

Ces caractéristiques de l'adaptation à la SEP justifient souvent que les capacités et ressources personnelles soient submergées par l'ampleur de la demande d'adaptation et engendrent des réactions pathologiques qui nécessiteront une intervention professionnelle en santé mentale. Les troubles de l'adaptation peuvent se décliner en deuil compliqué et en deuil pathologique, ce dernier rejoignant les troubles de santé mentale.

Le deuil compliqué est caractérisé par une perturbation du travail de deuil qui ne s'engage pas ou qui ne parvient pas à son terme. Le deuil pathologique est caractérisé par la survenue de troubles psychiatriques durant la période du deuil (de Montigny, 2002). Il est parfois nécessaire d'obtenir de l'aide professionnelle pour distinguer le normal du pathologique. Comme contribution originale, la figure 2 illustre par une courbe parallèle comment la psychopathologie peut être conçue comme un trouble de l'adaptation, l'exacerbation d'un processus normal.

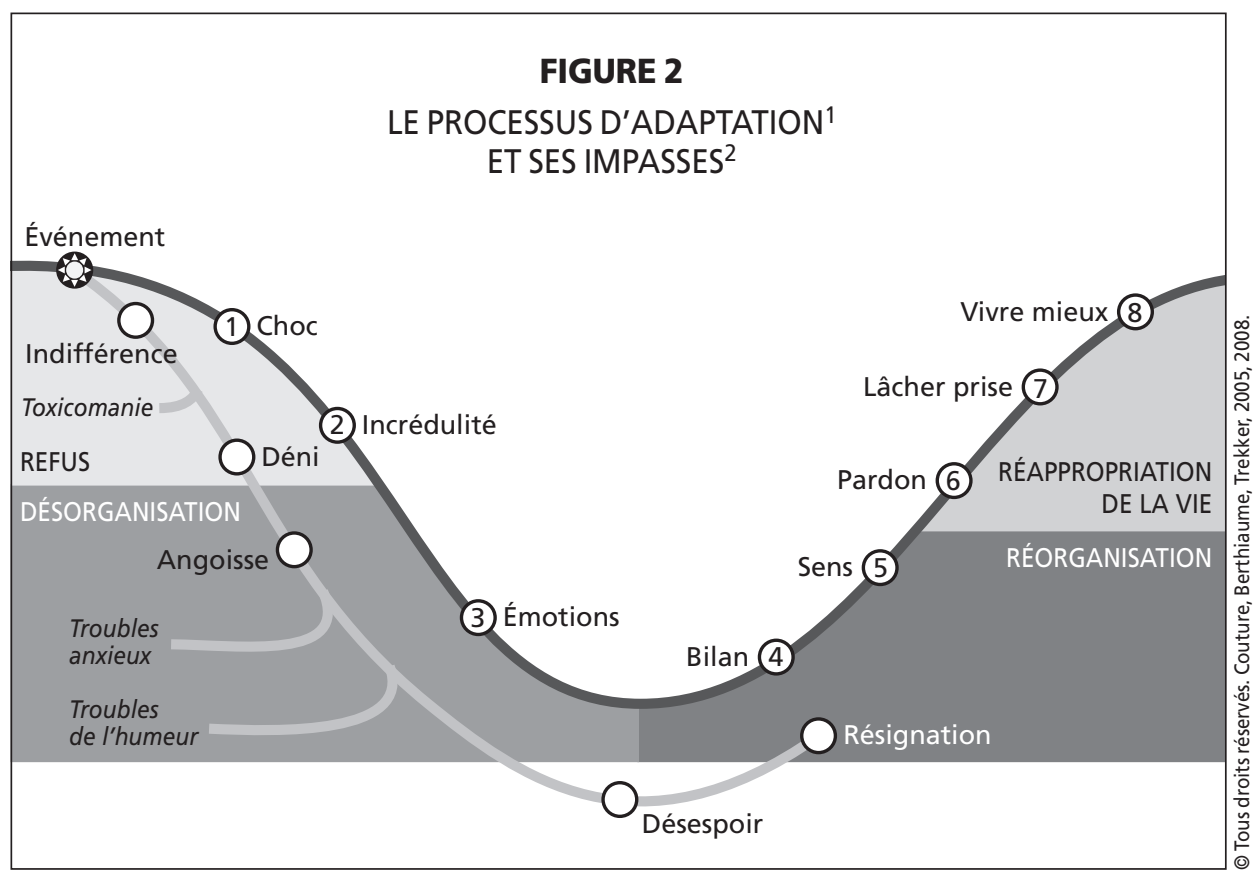

1. Inspiré de Monbourquette (1983); Lemieux (2002); St-Germain (1996).

2. Inspiré de Lacroix et Assal (1998); De Montigny (2002).

LES IMPASSES DE L'ADAPTATION :

\section{LE DEUIL COMPLIQUÉ}

Le deuil compliqué peut prendre différentes formes: deuil absent ou retardé, deuil intensifié, deuil inachevé. Le deuil compliqué mime en l'accentuant les étapes du processus d'adaptation normale. Pour se rétablir, il peut faire l'objet d'un soutien de type communautaire: proche ayant une bonne capacité d'écoute, groupe d'entraide, etc.

Le deuil peut être absent ou retardé. Il se manifeste alors par l'indifférence, c'est-àdire un détachement inapproprié dans sa durée ou son ampleur. L'angoisse en est un signal. Le déni est une forme active de fuite par la prise de risques indus et le recours à la pensée magique qui vont provoquer l'effondrement du mécanisme (Lacroix et Assal, 1998). Certains auteurs soulignent que l'absence de détresse peut être le fait de gens plus pragmatiques qui résolvent leur deuil sans débordement émotif sans que ce soit pathologique pour autant (Wortman et Silver, 1989).

Au contraire, le deuil peut être intensifié et envahissant. Les émotions prennent alors des proportions et des colorations qui enveniment le quotidien. Une gamme de réactions allant de l'angoisse au désespoir en passant par la culpabilité et la dévalorisation va entacher l'image et l'estime de soi. Il peut alors être utile de revisiter l'histoire des deuils anciens pour comprendre l'ampleur de la réaction actuelle.

Le deuil peut rester inachevé et se traduire par la résignation qui est une forme de soumission conduisant à la rumination, à l'amertume et à l'apitoiement. La personne malade se retire socialement et devient dépendante, jamais loin du désespoir. La résignation signifie qu'elle subit dès lors sa maladie comme un destin inexorable, s'en remettant entièrement aux soignants ou à son entourage (Lacroix et Assal, 1998).

\section{LES IMPASSES SÉVÈRES DE L'ADAPTATION: LE DEUIL PATHOLOGIQUE}

Le deuil est dit pathologique s'il engendre des troubles de santé mentale pouvant nécessiter une intervention professionnelle. L'évaluation et le diagnostic différentiel sont complexes, étant donné les recoupements entre les symptômes typiques de la SEP et ceux attribuables à la psychopathologie comme l'illustre la figure 3 .

\section{TOXICOMANIE}

L'abus de substances, que ce soit de l'alcool, de la drogue, des médicaments ou des activités compulsives comme le jeu, l'internet ou la sexualité servent à entretenir 


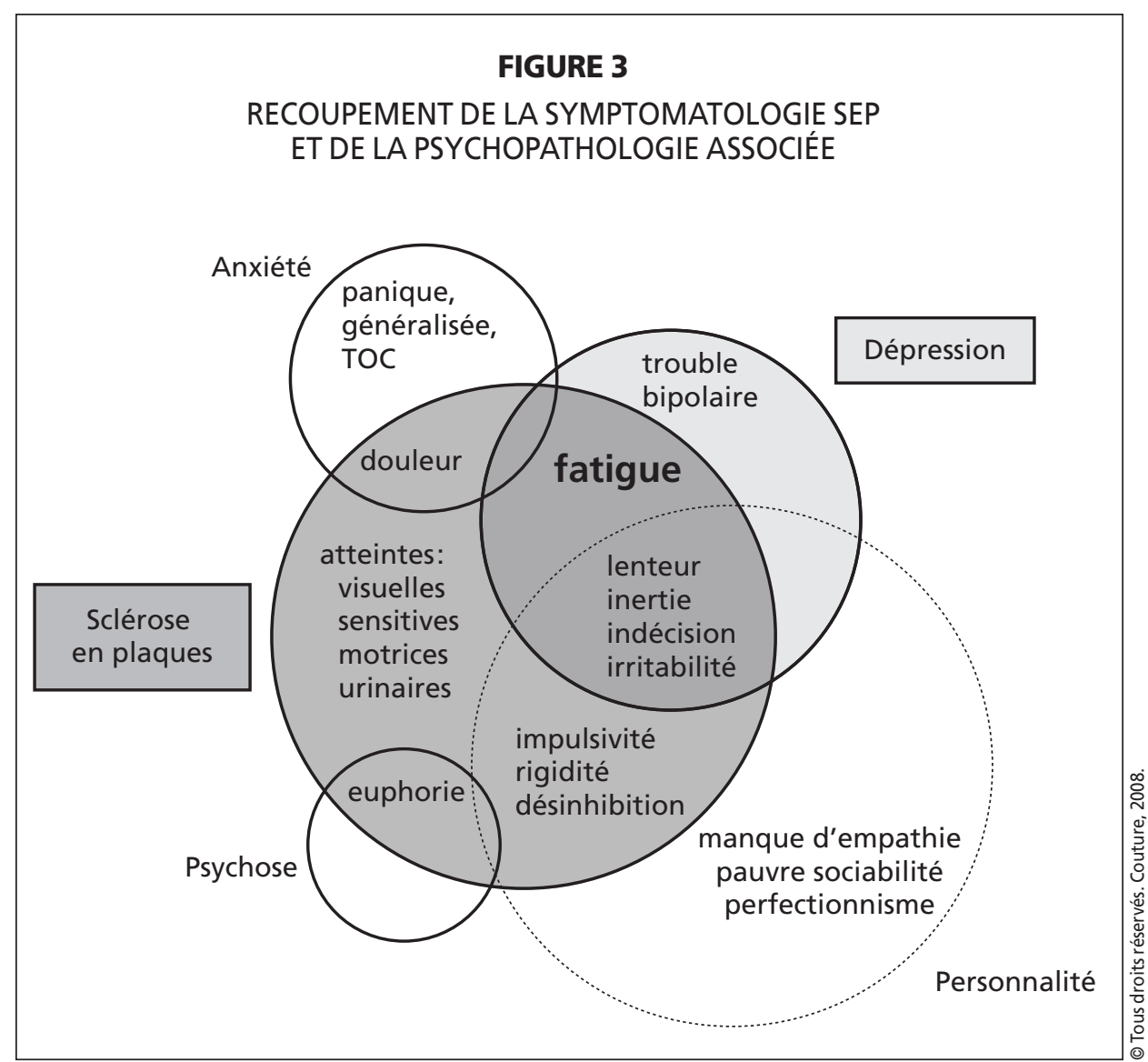

un écran pour éviter de faire face à la réalité. Ce faisant, il est courant que la personne s'enfonce davantage. Entre autres, l'alcoolisme est associé à plus de dépression et d'anxiété et augmente le risque suicidaire (Goldman Consensus Group, 2005; Korostil et Feinstein, 2007). Par ailleurs, le recours à la marijuana pour contrôler les spasmes, l'ataxie et la douleur est invoqué chez une minorité de personnes atteintes de SEP, généralement plus jeunes. La comorbidité psychiatrique est alors fréquente et le ralentissement du traitement de l'information, accentué (Ghaffar et Feinstein, 2008).

\section{TROUBLES ANXIEUX}

L'anxiété est diagnostiquée chez plus du tiers des personnes atteintes de SEP au cours de leur existence, qu'elle prenne une forme panique ou généralisée ou celle d'un trouble obsessionnel-compulsif. Les préoccupations sont souvent liées aux symptômes, l'incontinence par exemple, et à l'évolution de la maladie, comme la peur de finir en fauteuil roulant ou dans un centre d'hébergement. L'anxiété peut donc générer des scénarii du pire et engendrer des comportements d'évitement qui conduiront à l'isolement social.

\section{TROUBLES DE L'HUMEUR}

La dépression est fréquente chez les gens atteints de SEP. Au moins 50\% en seront affectés au cours de leur vie (Goldman
Consensus Group, 2005). Dans un échantillon fréquentant une clinique spécialisée de SEP, 79\% en présentent certains signes (Diaz-Olavarrieta et al., 1999). Le risque suicidaire est augmenté du double chez les gens atteints de SEP et explique 15\% des décès des patients fréquentant une clinique spécialisée (Turner et al., 2006).

Comment concevoir la résilience dans un tel contexte?

\section{LA RÉSILIENCE}

La résilience est un concept qui a gagné en popularité depuis les années 1970. Il fait référence à «la capacité à réussir à vivre et à se développer positivement de manière socialement acceptable en dépit du stress ou d'une adversité qui comporte normalement le risque grave d'une issue négative» (Cyrulnik, 2002, p. 8). Les recherches sur la résilience se sont jusqu'à présent plus souvent intéressées à la perspective développementale et aux enfants soumis à des conditions de vie difficiles (Luthar et al., 2000).

Selon certains auteurs, la résilience est le fait de caractéristiques personnelles spécifiques comme la régulation émotionnelle et la capacité d'attachement, l'intelligence, l'optimisme, l'altruisme, etc. (Charney, 2004). Dans le même sens, Fonagy (2004) pose quelques marqueurs de la résilience: cohérence, capacité réflexive, recherche efficace de soutien, sens de l'identité et de l'autonomie, capacité de faire la différence ente le rêve et la réalité. Or pour cet auteur et pour d'autres (Mikulincer et Shaver, 2007), la capacité réflexive, la mentalisation, s'acquiert au sein d'un attachement sécure, qui permet d'intégrer un regard positif sur soi et sur la vie, pouvant être développé précocement ou acquis par un cheminement thérapeutique. Pour d'autres auteurs, et sans que ce soit nécessairement antithétique avec la vision précédente, la résilience est le résultat d'un processus dynamique qui conduit à une adaptation positive malgré l'adversité (Luthar et al., 2000). Il peut s'agir de «la rencontre d'une personne signifiante [...] qui transfigure la souffrance... et fait germer le désir de s'en sortir»(Cyrulnik, 2004, p. 225). Que ce soit le fait de dispositions antérieures ou d'une rencontre significative, la question de donner un sens positif à l'expérience paraît centrale pour comprendre la capacité de résilience. Le détachement, le deuil, se ferait à l'aune de l'attachement?

\section{LA RECHERCHE DE SENS}

La question du sens est aussi déterminante dans la résolution du deuil (Neimeyer, 2000). Il semble qu'elle le soit de même dans l'adaptation à la maladie. Déjà, en 1987, le Dr Burnfield, un psychiatre atteint de SEP, soulignait l'importance de donner un sens à une expérience aussi absurde que la maladie pour en faire une occasion de croissance personnelle. Il faisait référence à Frankl, un autre psychiatre ayant traversé l'adversité des camps de concentration et qui y a survécu en se donnant la mission de témoigner de son expérience. La logothérapie qu'il a créée est expliquée dans plusieurs ouvrages phares (Frankl, 1985 [1959], 1970, 1973, 1993). Frankl estime que la quête de sens se fait à travers la conscience que l'homme développe de sa liberté face à ses conditionnements, en tenant compte de ses responsabilités. Pour lui, le bonheur ne constitue pas une fin en soi mais passe par la recherche d'une raison d'être heureux, à partir de laquelle le bonheur survient de soi. Le but de l'existence est de se réaliser, se dépasser, en se mettant au service d'une mission. Le sens ne peut être donné, il s'agirait alors de moralisation, il doit plutôt être découvert. Frankl explique que la volonté de signification revient apparemment à la saisie d'une "forme» au sens de la théorie de la gestalt. La souffrance devient donc une occasion parmi d'autres de régler un problème existentiel inhérent à la condition humaine.

\section{SENS ET RÉSILIENCE DANS LA SEP}

Des études récentes (Pakenham, 2007, 2008) viennent étayer cette théorisation du processus d'adaptation et de la résilience 
pour la SEP. Parmi un échantillon de près de quatre cents personnes atteintes et de plus de deux cents proches soignants, 53\% ne peuvent trouver un sens à leur maladie. Chez les autres, une analyse factorielle permet de faire émerger six façons principales de donner un sens qui ensemble expliquent un peu plus de $55 \%$ de la variance: donner un nouveau sens à sa vie $(24,77 \%)$, considérer la perspective spirituelle $(9,09 \%)$, faire des attributions causales $(6,5 \%)$, changer ses valeurs et ses priorités $(6,44 \%)$, accepter $(4,78 \%)$ et invoquer le hasard (4,06\%). Parmi les 44\% qui ont trouvé un sens, la dépression était moins présente et la satisfaction de vie plus grande chez les personnes qui avaient profité de la maladie pour redéfinir l'orientation de leur existence ou qui l'avaient acceptée. À l'inverse, la dépression était importante et l'état d'esprit positif faible chez celles qui n'y voyaient que l'effet du hasard. Les gens qui avaient changé leurs valeurs étaient anxieux, souvent en début de maladie, ce qui apparaissait à l'auteur comme une étape intermédiaire du processus d'adaptation. À noter que l'autonomie fonctionnelle constituait la variable qui prédisait le mieux l'adaptation; plus grande elle était, plus les gens étaient satisfaits de leur vie. Donc moins ils étaient affectés physiquement par la maladie, plus ils parvenaient à donner un sens positif au fait d'avoir la SEP.

Dans le même ordre d'idées, une autre étude établit un lien entre la dépression, l'affect positif, l'optimisme et la capacité de trouver des avantages à la SEP (Hart et al., 2008). Une thérapie téléphonique d'une durée de seize semaines, selon deux modalités thérapeutiques, cognitive et existentielle, permet d'observer des corrélations dans la fluctuation de ces variables. Peu importe la forme de thérapie, l'allégement de la dépression est associé à un plus grand optimisme et à un élargissement de la réponse teintée d'affect positif. Les auteurs considèrent ces facteurs comme des médiateurs aidant à entrevoir des bénéfices à leur expérience. Le modèle mathématique ne permet cependant pas d'établir une chronologie et encore moins une causalité entre les variables; elles apparaissent somme toute comme des vases communicants. Hart et ses collaborateurs estiment que l'amélioration de l'humeur et de l'attitude est une voie vers la résilience. Ils soulignent toutefois qu'ils ignorent si le changement d'attitude se traduit concrètement dans la vie quotidienne ou s'il ne demeure qu'une vision de l'esprit pour ceux qui le rapportent.

Qu'est-ce qui distingue ceux qui parviennent à une adaptation créative de ceux qui s'enlisent? Qu'est-ce qui caractérise ceux qui résolvent leur problème un à un de ceux qui sombrent dans le désespoir?

\section{SENS, RÉSILIENCE}

\section{ET RÉADAPTATION EN SEP}

La résilience rejoint la perspective du deuil résolu. Car l'adaptation est possible pour un peu plus du quart $(28 \%)$ d'un échantillon qui ne présente aucune manifestation psychopathologique malgré l'avancée de la maladie et près de la moitié qui évitera la dépression au cours de son existence (Joffe et al., 1987). Cette proportion semble correspondre aux données de Pakenham (Pakenham, 2007, 2008) pour ceux qui trouvent un sens positif à la SEP. Alors même que Meyer et Eckert (2002) croient le «deuil impossible», il semble donc que plus du tiers soit capable de résilience face à l'adversité et parvienne à faire de la maladie «un moyen pour acquérir de la maturité» (Burnfield, 1987). Au plan comportemental, il a été établi que la façon de faire face aux changements de vie sera associée à plus ou moins de dépression; la fuite et l'évitement émotif seront moins efficaces que la résolution de problèmes par exemple (Goldman Consensus Group, 2005; Wallin et al., 2006). Et comme les manifestations psychiatriques diminuent avec le temps, les chercheurs y voient l'indice du développement de stratégies efficaces avec l'avancée de la maladie (Beiske et al., 2008).

L'altruisme, la réciprocité et l'habileté à attirer et à utiliser le soutien social sont des caractéristiques de la résilience (Charney, 2004). L'altruisme et l'empathie sont aussi considérés comme des marqueurs de la compétence sociale. Or, certaines études mettent en évidence des traits de personnalité qui peuvent expliquer l'insatisfaction chronique qui caractérise certaines personnes atteintes de SEP et leur isolement social. Entre autres, la réduction de l'empathie et la perte de la sociabilité sont de ceux-là. Le perfectionnisme fait aussi partie du tableau (Benedict et al., 2001; Merkelbach et al., 2003).

Charney (2004) identifie certains circuits neuronaux comme vecteur important de la régulation émotionnelle et de la capacité de résolution de problèmes de haut niveau qui permettraient de s'extraire des réponses automatiques en attribuant une signification aux stimuli environnementaux. Or, le défaut de conscience et l'extraversion sont associés à l'atrophie cérébrale et pourraient expliquer certains changements de personnalité observables chez les personnes atteintes de SEP (Benedict et al., 2008). Il y a lieu de se demander si les atteintes cognitives associées à la SEP viennent entraver l'adaptation en affectant la base neuronale de la coopération sociale, minant ainsi la capacité réflexive et les habiletés relationnelles. Et si oui, comment intervenir?
Les recherches se font encore rares qui vérifient comment la résilience s'inscrit dans la vie de tous les jours des personnes handicapées par la SEP. Les données récentes vont dans le sens d'une amélioration des capacités ou à tout le moins de la qualité de vie des participants à un programme de réadaptation. Dans la logique d'une condition évolutive ayant des implications dans toutes les sphères de la vie, l'intervention se doit d'être multidisciplinaire et concertée (Khan, Pallant et al., 2008; Khan, Turner-Stokes et al., 2008).

Comment vivre avec la SEP et maintenir une qualité de vie? Le programme des maladies évolutives au Centre de réadaptation Lucie-Bruneau (CRLB) travaille en ce sens. Une approche interdisciplinaire soutient la recherche d'un nouvel équilibre entre la personne atteinte et son environnement. Les aménagements physiques et fonctionnels font l'objet d'interventions spécifiques en lien avec une démarche psychosociale où le bien-être psychologique, la gestion d'énergie et le soutien des proches sont concomitants.

L'activité de groupe est privilégiée, et ce, dès l'accueil dans le programme. Car les échanges entre pairs permettent l'entraide, brisent l'isolement, normalisent les diverses réactions en multipliant les exemples de façons de composer avec la maladie, offrant ainsi des points de vue alternatifs qui ouvrent des horizons. Apprendre à demander et accepter de l'aide, à reconnaître et à respecter ses propres besoins mais aussi ceux de ses proches sont d'autres avenues explorées par le programme des maladies évolutives du CRLB tout au long du séjour en réadaptation. Ultimement le programme permet à la personne atteinte non pas d'accepter la maladie mais de s'accepter avec la maladie. Les proches sont aussi intégrés à la démarche. Car ils ont aussi à se redéfinir face aux contraintes de la SEP et donc à entreprendre un processus d'adaptation parallèle à celui de la personne atteinte de SEP.

\section{VIVRE EN DÉSÉQUILIBRE}

L'activité «Vivre en déséquilibre» s'inscrit dans ce contexte et amorce la démarche de réadaptation. Elle vise la normalisation de la réponse d'adaptation et la gestion du stress. À travers la réflexion autour de l'adaptation, les participants, au nombre de 5 à 12 , vont mieux comprendre et accepter leurs réactions à la maladie et celles de leurs proches. L'acquisition d'informations pertinentes, l'autoévaluation et les échanges entre pairs sont favorisés au cours des huit semaines que dure l'activité. La gestion de l'énergie en lien avec la fatigue spécifique à la SEP et 
la gestion du stress, à cause de leur effet délétère sur la santé, sont deux thématiques importantes abordées parallèlement au processus d'adaptation. Pour créer une disposition physique, mentale et affective favorable aux échanges, pour expérimenter diverses formes de détente en lien avec les principes d'économie d'énergie enseignés ailleurs, pour minimiser l'impact du stress sur la santé, chaque rencontre débute par une période de relaxation. Le processus de résolution de problème peut aussi faire partie de l'approche éducative en lien avec la gestion du stress.

L'activité n'a pas encore donné lieu à une démarche évaluative formelle. Cependant, des observations cliniques et les témoignages des participants nous permettent d'ores et déjà de rendre compte du cheminement de certains. De plus, un outil maison offre la possibilité de faire un histogramme illustrant les étapes caractéristiques, ce qui permet à chacun de visualiser son avancée dans le processus. L'outil maison fournit une série d'énoncés dont plusieurs rejoignent l'étude de Pakenham (2007). La comparaison entre les choix de phrases effectués lors de la première et de la dernière semaine de l'activité indique l'amorce d'un cheminement et favorise un début de recherche de sens.

En quelque huit semaines, une usagère a ainsi progressé. Lors de la présentation initiale, elle racontait: "J'ai développé la SEP après le décès de mon mari que j'ai vécu très difficilement» et a choisi l'énoncé : «Je ne pourrai plus jamais séduire ou plaire». Quelques semaines plus tard, elle nuançait: «En fait, je ne voudrais imposer cela à personne ", pour réviser après quelque temps: "Je suis agréable à fréquenter. » Rougissant un peu lors de la dernière rencontre, elle a déclaré: «Je crois qu'un voisin s'intéresse à moi. »

Rechercher le sens, le plaisir et le contrôle dans le respect de ses énergies permet de retrouver l'espoir, celui qui porte vers l'avenir. Reprendre contact avec ses compétences et ses intérêts sont des moyens de se voir positivement dans l'œil de l'autre. Constater la résilience chez des pairs stimule la capacité d'y croire pour soi. En lui-même, le cheminement proposé par l'activité "Vivre en déséquilibre», en permettant l'auto-évaluation du processus d'adaptation et le soutien par les pairs, donne un sens à l'expérience et permet une avancée observable vers la réorganisation ${ }^{3}$.

\section{RECHERCHE DE SENS, DÉFIS ET ESPOIRS}

Il reste beaucoup à comprendre pour "souffler sur la braise de la résilience» (Cyrulnik, 2008). La SEP représente un champ d'investigation pertinent en regard de l'ampleur des défis à relever pour s'adapter. Faire preuve de résilience et vivre mieux avec la SEP demeurent des enjeux de taille lorsque l'épreuve est récurrente et que les capacités fonctionnelles et l'énergie diminuent. Des recherches seront nécessaires pour déterminer dans quelle mesure on peut compter sur l'amélioration des capacités relationnelles et comportementales de la personne présentant des limitations cognitives. Une étude récente laisse croire au potentiel de cette avenue (Benedict et al., 2000). Jusqu'à quel point et de quelle façon faut-il viser la compensation par les proches?

Il convient de se rappeler que vivre avec la SEP s'inscrit aussi dans un contexte social qui rend la tâche d'adaptation encore plus complexe. Lorsque son existence même témoigne de la face occultée de l'expérience humaine, qu'elle réintroduit la vulnérabilité, l'austérité et le tragique, qu'elle oblige à faire le deuil de soi tout en étant toujours là, qu'elle s'inscrit en faux contre le diktat de la performance, la SEP ne remet-elle pas en question sa place et son rôle dans la société? Le milieu de la réadaptation fournit un nouveau milieu d'appartenance, permet l'éclosion et la redéfinition d'une nouvelle norme sociale. Mais offrons-nous, ce faisant, un monde parallèle de référence dont les personnes atteintes ont du mal à s'extraire et qui confirme leur mise en marge? Ou, dans cette serre, un tuteur à la résilience et une occasion de répondre à certaines questions existentielles incontournables, qui serviront de ferment à l'émergence d'une société plus équilibrée et intégratrice de toutes ses forces vives? Et si oui, comme nous l'espérons, nous avons encore à préciser le soutien nécessaire à la personne atteinte de SEP et à ses proches pour favoriser l'émergence de cette résilience.

\section{Bibliographie}

ATCHLEY, R.C. (1982). "The aging self», Psychotherapy: Theory, Research, and Practice, vol. 19, p. 388-396.

BEISKE, A.G., E. SVENSSON, B. CZUJKOE, E.D. PEDERSENA, J.H. AARSETHF et K.M. MYHRF (2008). "Depression and anxiety among multiple sclerosis patients ", European Journal of Neurology, vol. 15, p. 239-245.

BENEDICT, R.H.B., S. HUSSEIN, J. ENGLERT, M.G. DWYER, N. ABDELRAHMAN, J.L. COX et al. (2008). "Cortical atrophy and personality in multiple sclerosis », Neuropsychology, vol. 22, p. 432-441.

BENEDICT, R.H.B., R.L. PRIORE, C. MILLER, F. MUNSCHAUER et L. JACOBS (2001). "Personality disorder in multiple sclerosis correlates with cognitive impairment», Journal of Neuropsychiatry and Clinical Neurosciences, vol. 13, p. 70.
BENEDICT, R.H.B., A. SHAPIRO, R. PRIORE, C. MILLER, F. MUNSCHAUER et L. JACOBS (2000). "Neuropsychological counseling improves social behavior in cognitively-impaired multiple sclerosis patients », Multiple Sclerosis, vol. 6, p. 391.

BOËRIO, D., J.P. LEFAUCHEUR, J.Y. HOGREL et A. CRÉANGE (2006). "Physiopathologie et traitement de la fatigue dans la sclérose en plaques ", Revue neurologique, vol. 162, p. 311-320.

BONANNO, G.A., A. PAPA, K. LALANDE, M. WESTPHAL et K. COIFMAN (2004). "The importance of being flexible», Psychological Science, vol. 15, p. 482-487.

BULJEVAC, D., W.C.J. HOP, W. REEDEKER, A. JANSSENS, F.G.A. VAN DER MECHE, P.A. VAN DOORN et al. (2003). "Self reported stressful life events and exacerbations in multiple sclerosis: Prospective study », British Medical Journal, vol. 327, p. 646.

BURNFIELD, A. (1987). "La sclérose en plaques: un moyen d'acquérir de la maturité? », SP Québec.

CHARNEY, D.S. (2004). «Psychobiological mechanisms of resilience and vulnerability implications for successful adaptation to extreme stress », American Journal of Psychiatry, vol. 161, p. 195-216.

CHWASTIAK, L.A. et D.M. EHDE (2007). "Psychiatric issues in multiple sclerosis", The Psychiatric Clinics of North America, vol. 30, p. 803-817.

COMTE-SPONVILLE, A. (2006). L'esprit de l'athéisme, Introduction à une spiritualité sans Dieu, Paris, Albin Michel.

CYRULNIK, B. (2002). Un merveilleux malheur, Paris, Odile Jacob.

CYRULNIK, B. (2004). Les vilains petits canards, Paris, Odile Jacob.

CYRULNIK, B. (2008). «Résilience, pratiques d'intervention», conférence donnée lors du congrès «Résilience, intervention en contexte d'adaptation et de réadaptation ", Montréal, 25 et 26 février 2008.

DE MONTIGNY, J. (2002). "Quand le deuil se complique », Psychologie Québec, vol. 19, p. $19-22$.

DESJARDINS, M. (2002). Le jardin d'ombre, La poétique et la politique de la rééducation sociale, Québec, Presses de l'Université du Québec.

DIAZ-OLAVARRIETA, C., J.L. CUMMINGS, J. VELAZQUEZ et C. GARCIA DE AL CADENA (1999). "Neuropsychiatric manifestations of multiple sclerosis ", Journal of Neuropsychiatry and Clinical Neurosiences, vol. 11, p. 51-57.

FONAGY, P. (2004). Théorie de l'attachement et psychanalyse, Paris, Érès.

FRANKL, V.E. (1985 [1959]). Man's Search for Meaning, Boston, Pocket Books.

FRANKL, V.E. (1970). La psychothérapie et son image de l'homme, Paris, Éditions Resma.

FRANKL, V.E. (1973). The Doctor and the Soul: From Psychotherapy to Logotherapy, New York, Vintage Books. 
FRANKL, V.E. (1993). Raisons de vivre, Genève, Éditions du Tricorne.

FREUD, S. (1986). «Deuil et mélancolie», dans Métapsychologie, Paris, Gallimard, p. $145-171$.

GHAFFAR, O. et A. FEINSTEIN (2008). "Multiple sclerosis and cannabis ", Neurology, vol. 71, p. 164-169.

GOLDMAN CONSENSUS GROUP (2005). "The Goldman Consensus statement on depression in multiple sclerosis", Multiple Sclerosis, vol. 11, p. 328-337.

HART, S.L., L. VELLA et D.C. MOHR (2008). "Relationships among depressive symptoms, benefit-finding, optimism, and positive affect in multiple sclerosis patients after psychotherapy for depression », Health Psychology, vol. 27, p. 230-238.

HÉTU, J.L. (1989). Psychologie du mourir et du deuil, Montréal, Éditions du Méridien.

JOFFE, R.T., G.P. LIPPERT, T.A. GRAY, G. SAWA et Z. HORVATH (1987). "Mood disorder and multiple sclerosis", Archives of Neurology, vol. 44, p. 376-378.

JOLLIEN, A. (2002). Le métier d'homme, Paris, Seuil

KALB, R.C. (2004). Multiple Sclerosis: The Questions You Have, The Answers You Need, New York, Demos Medical Publishing.

KEIRSE, M. (2000). Faire son deuil, vivre son chagrin, Bruxelles, DeBoeck \& Belin.

KHAN, F., J.F. PALLANT, C. BRAND et T.J. KILPATRICK (2008). " Effectiveness of rehabilitation intervention in persons with multiple sclerosis: A randomized controlled trial ", Journal of Neurology, Neurosurgery and Psychiatry, vol. 79, p. 1230-1235.

KHAN, F., L. TURNER-STOKES, L. NG et T. KILPATRICK (2008). « Multidisciplinary rehabilitation for adults with multiple sclerosis ", Journal of Neurology, Neurosurgery and Psychiatry, vol. 79, p. 114.

KOROSTIL, M. et A. FEINSTEIN (2007). "Anxiety disorders and their clinical correlates in multiple sclerosis patients ", Multiple Sclerosis, vol. 13, p. 67-72.
LACROIX, A. et J.P. ASSAL (1998). L'éducation thérapeutique des patients, Nouvelles approches de la maladie chronique, Paris, Vigot.

LEFEBVRE, H. et M.J. LEVERT (2005). "Traumatisme craniocérébral: de la souffrance à la résilience », Frontières, vol. 17, p. $77-85$

LEMIEUX, M. (2002). "Le deuil: incontournable réalité méconnue de la vie», Psychologie Québec, vol. 19, p. 12-15.

LUTHAR, S.S., D. CICCHETTI et B. BECKER (2000). "The construct of resilience: A critical evaluation and guidelines for future work ", Child Development, vol. 71, p. 543-562.

MERKELBACH, S., J. KONIG et H. SITTINGER (2003). "Personality traits in multiple sclerosis (MS) patients with and without fatigue experience», Acta Neurologica Scandinavica, vol. 107, p. 195-201.

MEYER, C. et F. ECKERT (2002). «L'impossible deuil», Journal de réadaptation médicale, vol. 22, p. 110-114.

MIKULINCER, M. et P.R. SHAVER (2007). Attachment in Adulthood: Structure, Dynamics, and Change, New York, The Guilford Press.

MONBOURQUETTE, J. (1994). Aimer, perdre et grandir, Ottawa, Novalis.

MONBOURQUETTE, J. (2007). Comment pardonner?, Coffragants.

NEIMEYER, R.A. (2000). "Searching for the meaning of meaning: Grief therapy and the process of reconstruction", Death Studies, vol. 24, p. 541-558.

PAKENHAM, K.I. (2007). "Making sense of multiple sclerosis ", Rehabilitation Psychology, vol. 52, p. 380-389.

PAKENHAM, K.I. (2008). «Making sense of illness or disability: The nature of sense making in multiple sclerosis (MS) », Journal of Health Psychology, vol. 13, p. 93-105.

PITZELE, S. (2000). Plus jamais seuls, Vivre avec une maladie chronique, Montréal, Éditions Logiques.
ROGERS, J.M. et P.K. PANEGYRES (2007). "Cognitive impairment in multiple sclerosis: Evidence-based analysis and recommendations ", Journal of Clinical Neuroscience, vol. 14, p. 919-927.

SAINT-CHARLES， D. (1998). "La reconstruction identitaire des "prochessoignants" des personnes traumatisées craniocérébrales», dans J.C. KALUBI, B. MICHALLET, N., KORNER-BITENSKY et S. TÉTREAULT (dir.), Innovations, apprentissages et réadaptation en déficience physique, $2^{\mathrm{e}}$ éd., Montréal, Isabelle Quentin éditeur, p. 97-103.

SAINT-GERMAIN, D. (1996). Pour t'aider à mieux vivre une perte, Port-Cartier, Québec, Centre de santé et de services sociaux de Port-Cartier.

SOCIÉTÉ CANADIENNE DE SCLÉROSE EN PLAQUES (2009). "À propos de la SP », en ligne, $<$ http://mssociety.ca/fr/informations/default.htm $>$, consulté le 2009-11-01

TREMBLAY, M. (2001). L'adaptation humaine, Un processus biopsychosocial à découvrir, Montréal, Saint-Martin.

TURNER, A.P., R.M. WILLIAMS, J.D. BOWEN, D.R. KIVLAHAN et J.K. HASELKORN (2006). "Suicidal ideation in multiple sclerosis ", Archives of Physical Medicine and Rehabilitation, vol. 87, p. $1073-1078$

WALLIN, M.T., J.A. WILKEN, A.P. TURNER, R.M. WILLIAMS et R. KANE (2006) «Depression and multiple sclerosis: Review of a lethal combination », Journal of Rehabilitation Research and Development, vol. 43, p. 45-62.

WORTMAN, C.B. et R.C. SILVER (1989) "The myths of coping with loss », Journal of Consulting and Clinical Psychology, vol. 57, p. 349-357.

\section{Notes}

1. Voir à ce sujet, l'article sur l'expression musicale dans le présent numéro.

2. Titre à la une de la revue L'actualité (juillet 2008).

3. Une vidéo a été produite au CRLB par des usagers du programme en 2000 . 